Proceedings of the

International Geometry Center

Vol. 12 , no. 3 (2019) pp. $69-77$

\title{
Coarse equivalences of functorial constructions
}

\author{
Mykhailo Romanskyi
}

\begin{abstract}
We consider the question of coarse equivalence of some functorial constructions (in particular, symmetric powers, hypersymmetric powers) in the category of metric spaces.
\end{abstract}

Анотація. Груба геометрія займається вивченням властивостей метричних просторів "в нескінченності" (див. [3]). Однією з важливих загальних задач грубої геометрії $\epsilon$ класифікація метричних просторів з точністю до грубої еквівалентності. Так в [6], було доведено, що гіперпростори cс $\left(\mathbb{R}^{n}\right)$, exp $\mathbb{R}^{n}$ та ехр ${ }^{c} \mathbb{R}^{n}$ не є грубо еквівалентними. В [7] доведено ізоморфність джойна $\mathbb{R}^{n} * \mathbb{R}_{+}$та півпростору $\mathbb{R}_{+}^{n+1}$ в асимптотичній категорії $\mathcal{A}$.

$\mathrm{B}$ цій статті показано, що гіперпростори $\exp _{3} \mathbb{R}_{+}, \exp _{3} \mathbb{R}$, симетричні степені $S P^{3} \mathbb{R}_{+}, S P^{3} \mathbb{R}$ та простір $\mathbb{R}_{+}^{3}$ ліпшицево еквівалентні (теореми $3.1,3.2)$. Крім того доведено, що простори $\mathbb{R}_{+}^{3}$ та $P_{2}(\mathbb{R})$ не $\epsilon$ грубо еквівалентними.

Одним з основних результатів є теорема 3.7 , про біліпшицеву еквівалентність гіперпростору $\exp _{2} \mathbb{R}^{m}$ та $\mathbb{R}^{m} \times$ Cone $\left(\mathbb{R} P^{m-1}\right)$. Ї̈̈ можна вважати асимптотичним аналогом одного результату Шорі [4].

В статті [1] означено конус $C X$ і надбудову $S X$ в асимптотичних категоріях для кожного метричного простору. У теоремі 3.12 доведено, що конус $C \mathbb{R}_{+}$і надбудова $S \mathbb{R}_{+}$не є грубо еквівалентні.

The author is indebted to Mykhailo Zarichnyi for valuable discussions.

Keywords: coarse equivalence, hypersymmetric powers, cone 


\section{INTRODUCTION}

Coarse geometry deals with properties of metric spaces (and more general coarse spaces) "at infinity" (see, e.g., [1], [3]).

One of the main general problems in coarse geometry is that of classification of spaces up to coarse equivalence.

A. Dranishnikov [1] introduced certain functorial constructions in the asymptotic categories. In particular, he considered the products, joins, cones, and spaces of probability measures. Several results concerning geometric properties of these spaces are also obtained in $[6,7]$.

We provide some necessary definitions.

For every metric space $X$ we denote by $\exp X$ the set of all nonempty compact subsets of a metrizable space $X$. Any admissiblle metric $d$ on $X$ induces the Hausdorff metric $d_{H}$ on $\exp X$ :

$$
d_{H}(A, B)=\inf \left\{\varepsilon>0 \mid A \subset O_{\varepsilon}(B), B \subset O_{\varepsilon}(A)\right\} .
$$

For every $n \in \mathbb{N}$ let $\exp _{n} X$ be the subspace of $\exp X$ consisting of all nonempty sets of cardinality $\leqslant n$. E. V. Shchepin [5] called the space $\exp _{n} X$ the $n$th hypersymmetric power of $X$. We will denote by $\exp ^{c} X$ the subspace of $\exp X$ consisting of all subcontinua of $X$.

It was proved in [6] that the hyperspaces cc $\left(\mathbb{R}^{n}\right)$, exp $\mathbb{R}^{n}$ and $\exp ^{c} \mathbb{R}^{n}$ are not coarsely equivalent. Also in [7] it was established that the join $\mathbb{R}^{n} * \mathbb{R}_{+}$and half-space $\mathbb{R}_{+}^{n+1}$ are isomorphic in the asymptotic category $\mathcal{A}$.

In this note we show that the hyperspaces $\exp _{3} \mathbb{R}_{+}$, $\exp _{3} \mathbb{R}$, symmetric powers $S P^{3} \mathbb{R}_{+}, S P^{3} \mathbb{R}$ and the space $\mathbb{R}_{+}^{3}$ are Lipschitz equivalent. In addition, we will prove that the spaces $\mathbb{R}_{+}^{3}$ and $P_{2}(\mathbb{R})$ are not coarsely equivalent. Here $P_{2}(X)$ is the subspace of the space of probability measures whose support consists of at most 2 points.

One of our main results is Theorem 3.7 on Lipschitz equivalence of the hypersymmetric power $\exp _{2} \mathbb{R}^{m}$ and $\mathbb{R}^{m} \times$ Cone $\left(\mathbb{R} P^{m-1}\right)$. This theorem can be considered as an asymptotic counterpart of one Schori's result [4].

\section{Preliminaries}

Let $\sim$ be the equivalence relation on $X^{n}$ defined by the condition:

$$
\left(x_{1}, \ldots, x_{n}\right) \sim\left(y_{1}, \ldots, y_{n}\right)
$$

if and only if there is a bijection $\sigma$ of $\{1, \ldots, n\}$ such that $x_{i}=y_{\sigma(i)}$ for all $i=1, \ldots, n$. The orbit space of this relation on $X^{n}$ is called the $n$th symmetric power of $X$ and is denoted by $S P^{n}(X)$. The equivalence class of $\sim$ containing $\left(x_{1}, \ldots, x_{n}\right)$ is denoted by $\left[x_{1}, \ldots, x_{n}\right]$. The support of $x=\left[x_{1}, \ldots, x_{n}\right] \in S P^{n}(X)$ is the set

$$
\operatorname{supp}(x)=\left\{x_{1}, \ldots, x_{n}\right\} \in \exp _{n} X .
$$


If $(X, d)$ is a metric space, then it is known that the following function $\hat{d}$ is a metric on $S P^{n}(X)$ :

$$
\hat{d}\left(\left[x_{1}, \ldots, x_{n}\right],\left[y_{1}, \ldots, y_{n}\right]\right)=\min _{\sigma} \max _{i} d\left(x_{i}, y_{\sigma(i)}\right) .
$$

A map $f:(X, d) \rightarrow(Y, \rho)$ is asymptotically Lipschitz, if there exist $\lambda>0$ and $s \geqslant 0$ such that

$$
\rho(f(x), f(y)) \leqslant \lambda d(x, y)+s, \quad x, y \in X .
$$

A map $f:(X, d) \rightarrow(Y, \rho)$ is called a bi-Lipschitz embedding, if there exists $\lambda>0$ such that

$$
\frac{1}{\lambda} d(x, y) \leqslant \rho(f(x), f(y)) \leqslant \lambda d(x, y), \quad x, y \in X .
$$

A map $f:(X, d) \rightarrow(Y, \rho)$ is called a coarse embedding if there exist non-decreasing functions $\varphi_{1}, \varphi_{2}:[0, \infty) \rightarrow[0, \infty)$ such that

$$
\varphi_{1}(d(x, y)) \leqslant \rho(f(x), f(y)) \leqslant \varphi_{2}(d(x, y)), \quad x, y \in X .
$$

In the case of geodesic metric spaces, every coarse uniform map is asymptotically Lipschitz, [1].

Let $D>0$. Recall that a subset $A$ of a metric space $X$ is called $D$ discrete, if $d(x, y) \geqslant D$ for all $x, y \in X, x \neq y$.

\section{MAin RESUlt}

Theorem 3.1. The hyperspace $\exp _{3} \mathbb{R}_{+}$, the symmetric power $S P^{3} \mathbb{R}_{+}$, and the space $\mathbb{R}_{+}^{3}$ are mutually Lipschitz equivalent.

Proof. Every point $[a, b, c] \in S P^{3} \mathbb{R}_{+}$can be written as $(a, b, c)$, where $a \leqslant b \leqslant c$. Then, representing $S P^{3} \mathbb{R}_{+}$in the Cartesian coordinate system we obtain the cone, Cone $\left(B^{2}\right)$. In the spherical coordinate system we see that

$$
S P^{3} \mathbb{R}_{+}=\left\{(r, \varphi, \theta) \mid 0 \leqslant r<+\infty, \frac{\pi}{4} \leqslant \varphi \leqslant \frac{\pi}{2}, 0 \leqslant \theta \leqslant \frac{\pi}{4}\right\},
$$

and the map $f: S P^{3} \mathbb{R}_{+} \rightarrow \mathbb{R}_{+}^{3}, f(r, \varphi, \theta)=\left(r, 2 \varphi-\frac{\pi}{2}, 2 \theta\right)$, is Lipschitz with constant $2 \sqrt{2}$.

Remark that the map $f^{-1}: \mathbb{R}_{+}^{3} \rightarrow S P^{3} \mathbb{R}_{+}$is given by the formula $f^{-1}(r, \varphi, \theta)=\left(r, \frac{\varphi}{2}+\frac{\pi}{4}, 2 \frac{\theta}{2}\right)$. Clearly, $f^{-1}$ is Lipschitz with constant $\frac{\sqrt{2}}{4}$.

Let $\sim$ be the equivalence relation on $S P^{3} \mathbb{R}_{+}$given by the condition

$$
[a, a, b] \sim[a, b, b] .
$$

The hyperspace $\exp _{3} \mathbb{R}_{+}$is the quotient space of the space $S P^{3} \mathbb{R}_{+}$with respect to this equivalence relation. This quotient space is nothing but the cone Cone $\left(B^{2}\right)$, which is known to be Lipschitz equivalent to $\mathbb{R}_{+}^{3}$. 
One can similarly prove that the symmetric power $S P^{k} \mathbb{R}_{+}$is Lipschitz equivalent to $\mathbb{R}_{+}^{k}$.

Theorem 3.2. The hypersymmetric power $\exp _{3} \mathbb{R}$, symmetric power $S P^{3} \mathbb{R}$ and $\mathbb{R}_{+}^{3}$ are Lipschitz equivalent.

Proof. Similarly to the proof of Theorem 3.1 we note that for any $[a, b, c] \in$ $S P^{3} \mathbb{R}$ one may assume, without loss of generality, that $a \leqslant b \leqslant c$, and we then identify this point with $(a, b, c)$. This allows us to represent $S P^{3} \mathbb{R}$ in the Cartesian coordinate system. The obtained space is easily seen to be Lipschitz equivalent to $\mathbb{R}_{+}^{3}$.

In order to obtain $\exp _{3} \mathbb{R}$ we have to make the identification

$$
[a, a, b] \sim[a, b, b]
$$

в $S P^{3} \mathbb{R}$. After this identification, the obtained space is Lipschitz equivalent to $\mathbb{R}_{+}^{3}$.

Theorems 3.1 and 3.2 imply the following.

Corollary 3.3. The hypersymmetric powers $\exp _{3} \mathbb{R}_{+}$, $\exp _{3} \mathbb{R}$, symmetric powers $S P^{3} \mathbb{R}_{+}, S P^{3} \mathbb{R}$ and $\mathbb{R}_{+}^{3}$ are Lipschitz equivalent.

The cone Cone $(X)$ over a compact metric space $(X, d)$ is the quotient space of the product $\left(X \times \mathbb{R}_{+}\right) / \sim$, where the equivalence relation $\sim$ is given by the condition $(x, 0) \sim(y, 0), x, y \in X$. If, in addition, $\operatorname{diam}(X) \leqslant 2$, then the metric $\hat{d}$ on Cone $(X)$ can be given by the formula:

$$
\hat{d}((x, t),(y, s))=\min \{t, s\} d(x, y)+|t-s| .
$$

Lemma 3.4. If compact metric spaces $(X, d)$ and $(Y, \rho)$ are Lipschitz equivalent, then the metric spaces $\operatorname{Cone}(X)$ and $\operatorname{Cone}(Y)$ are also Lipschitz equivalent.

Proof. Since the metric spaces $(X, d)$ and $(Y, \rho)$ are Lipschitz equivalent, there exists a bijective map $f:(X, d) \rightarrow(Y, \rho)$ such that

$$
\frac{1}{\lambda} d(x, y) \leqslant \rho(f(x), f(y)) \leqslant \lambda d(x, y), \quad x, y \in X
$$

for some $\lambda \geqslant 1$.

Define a bijective map $g:(\operatorname{Cone}(X), \hat{d}) \rightarrow(\operatorname{Cone}(Y), \hat{\rho})$ by the formula $g(x, t)=(f(x), t)$ and prove that this map is bi-Lipschitz. We have

$$
\begin{aligned}
\hat{\rho}\left(g\left(x_{1}, t\right), g\left(x_{2}, s\right)\right) & =\hat{\rho}\left(\left(f\left(x_{1}\right), t\right), f\left(\left(x_{2}\right), s\right)\right) \\
& =\min \{t, s\} \cdot \rho\left(f\left(x_{1}\right), f\left(x_{2}\right)\right)+|t-s| \\
& \leqslant \min \{t, s\} \cdot \lambda \cdot d\left(x_{1}, x_{2}\right)+|t-s|
\end{aligned}
$$




$$
\begin{aligned}
& \leqslant \lambda \cdot\left(\min \{t, s\} \cdot d\left(x_{1}, x_{2}\right)+|t-s|\right) \\
& =\lambda \cdot \hat{d}\left(\left(x_{1}, t\right),\left(x_{2}, s\right)\right)
\end{aligned}
$$

On the other hand,

$$
\begin{aligned}
\hat{\rho}\left(g\left(x_{1}, t\right), g\left(x_{2}, s\right)\right) & =\hat{\rho}\left(\left(f\left(x_{1}\right), t\right), f\left(\left(x_{2}\right), s\right)\right) \\
& =\min \{t, s\} \cdot \rho\left(f\left(x_{1}\right), f\left(x_{2}\right)\right)+|t-s| \\
& \geqslant \min \{t, s\} \cdot \frac{1}{\lambda} \cdot d\left(x_{1}, x_{2}\right)+|t-s| \\
& \geqslant \frac{1}{\lambda} \cdot\left(\min \{t, s\} \cdot d\left(x_{1}, x_{2}\right)+|t-s|\right) \\
& =\frac{1}{\lambda} \cdot \hat{d}\left(\left(x_{1}, t\right),\left(x_{2}, s\right)\right) .
\end{aligned}
$$

Lemma is proved.

Lemma 3.5. The hemisphere $\mathrm{S}_{+}^{n}$ and the cube $\mathrm{I}^{n}$ are Lipschitz equivalent.

Proof. Define $f: \mathrm{I}^{n} \rightarrow \mathrm{S}_{+}^{n}$ as the composition of two bi-Lipschitz maps, $f=g \circ \varphi$, where $g: \mathrm{B}^{n} \rightarrow \mathrm{S}_{+}^{n}$ is the stereographic projection, and the map $\varphi: \mathrm{I}^{n} \rightarrow \mathrm{B}^{n}$ is given by the formula:

$$
\varphi\left(x_{1}, x_{2}, \ldots, x_{n}\right)=\frac{\left(x_{1}, x_{2}, \ldots, x_{n}\right)}{\left\|\left(y_{1}, y_{2}, \ldots, y_{n}\right)\right\|},
$$

where $\left(y_{1}, y_{2}, \ldots, y_{n}\right) \in \operatorname{Bd}\left(\mathrm{I}^{n}\right)$ is such that $\left(y_{1}, y_{2}, \ldots, y_{n}\right)=k \cdot\left(x_{1}, x_{2}, \ldots, x_{n}\right)$ for some $k$.

The Lipschitz constant for $\varphi$ is $\lambda_{1}=2$. Also it is known that the constant $\lambda_{2}$ for the stereographic projection $g$ equals 2 . Therefore, $f$ is bi-Lipschitz with constant $\lambda=\lambda_{1} \cdot \lambda_{2}=4$.

By Lemmas 3.4 and 3.5 taking into account that Cone $\left(S_{+}^{n}\right) \simeq \mathbb{R}_{+}^{n+1}$ we obtain the following result.

Corollary 3.6. The cone Cone $\left(\mathrm{I}^{n}\right)$ and $\mathbb{R}_{+}^{n+1}$ are Lipschitz equivalent.

Theorem 3.7. The hyperspace $\exp _{2} \mathbb{R}^{m}$ is Lipschitz equivalent to

$$
\mathbb{R}^{m} \times \text { Cone }\left(\mathbb{R} P^{m-1}\right)
$$

Proof. Define $f:\left(\exp _{2} \mathbb{R}^{m}, d_{H}\right) \rightarrow\left(\mathbb{R}^{m} \times\right.$ Cone $\left.\left(\mathbb{R} P^{m-1}\right), \rho\right)$ by the formula

$$
f(x, y)=\left(\frac{x+y}{2},\left(\frac{x-y}{\|x-y\|},\|x-y\|\right)\right) ; x, y \in \mathbb{R}^{m} .
$$

We first prove that $f^{-1}$ is Lipschitz. Indeed,

$$
\begin{gathered}
d_{H}\left(\left(x_{1}, y_{1}\right),\left(x_{2}, y_{2}\right)\right) \leqslant d_{\mathbb{R} P^{m-1}}\left(\frac{x_{1}-y_{1}}{\left\|x_{1}-y_{1}\right\|}, \frac{x_{2}-y_{2}}{\left\|x_{2}-y_{2}\right\|}\right) \cdot \min \left\{\left\|x_{1}-y_{1}\right\|,\left\|x_{2}-y_{2}\right\|\right\} \\
+\left|\left\|x_{1}-y_{1}\right\|-\left\|x_{2}-y_{2}\right\|\right|+d_{\mathbb{R}^{m}}\left(\frac{x_{1}+y_{1}}{2}, \frac{x_{2}+y_{2}}{2}\right)
\end{gathered}
$$




$$
\begin{aligned}
& \leqslant 2 \max \left\{d_{\mathbb{R} P^{m-1}}\left(\frac{x_{1}-y_{1}}{\left\|x_{1}-y_{1}\right\|}, \frac{x_{2}-y_{2}}{\left\|x_{2}-y_{2}\right\|}\right) \cdot \min \left\{\left\|x_{1}-y_{1}\right\|,\left\|x_{2}-y_{2}\right\|\right\}\right. \\
& \left.\quad+\left|\left\|x_{1}-y_{1}\right\|-\left\|x_{2}-y_{2}\right\|\right|, d_{\mathbb{R}^{m}}\left(\frac{x_{1}+y_{1}}{2}, \frac{x_{2}+y_{2}}{2}\right)\right\} \\
& =2 \rho\left(\left(\frac{x_{1}+y_{1}}{2},\left(\frac{x_{1}-y_{1}}{\left\|x_{1}-y_{1}\right\|},\left\|x_{1}-y_{1}\right\|\right)\right),\left(\frac{x_{2}+y_{2}}{2},\left(\frac{x_{2}-y_{2}}{\left\|x_{2}-y_{2}\right\|},\left\|x_{2}-y_{2}\right\|\right)\right)\right) \\
& =2 \rho\left(f\left(x_{1}, y_{1}\right), f\left(x_{2}, y_{2}\right)\right) .
\end{aligned}
$$

Now we prove that $f$ is Lipschitz. We have

$$
\begin{aligned}
& \rho\left(\left(\frac{x_{1}+y_{1}}{2},\left(\frac{x_{1}-y_{1}}{\left\|x_{1}-y_{1}\right\|}, \|\right.\right.\right.\left.\left.\left.x_{1}-y_{1} \|\right)\right),\left(\frac{x_{2}+y_{2}}{2},\left(\frac{x_{2}-y_{2}}{\left\|x_{2}-y_{2}\right\|},\left\|x_{2}-y_{2}\right\|\right)\right)\right)= \\
&=\max \{ d_{\mathbb{R}^{m}}\left(\frac{x_{1}+y_{1}}{2}, \frac{x_{2}+y_{2}}{2}\right), \\
&\left.\hat{d}\left(\left(\frac{x_{1}-y_{1}}{\left\|x_{1}-y_{1}\right\|},\left\|x_{1}-y_{1}\right\|\right),\left(\frac{x_{2}-y_{2}}{\left\|x_{2}-y_{2}\right\|},\left\|x_{2}-y_{2}\right\|\right)\right)\right\}
\end{aligned}
$$

and

$$
\begin{aligned}
& \hat{d}\left(\left(\frac{x_{1}-y_{1}}{\left\|x_{1}-y_{1}\right\|},\left\|x_{1}-y_{1}\right\|\right),\left(\frac{x_{2}-y_{2}}{\left\|x_{2}-y_{2}\right\|},\left\|x_{2}-y_{2}\right\|\right)\right)= \\
& =d_{\mathbb{R} P^{m-1}}\left(\frac{x_{1}-y_{1}}{\left\|x_{1}-y_{1}\right\|}, \frac{x_{2}-y_{2}}{\left\|x_{2}-y_{2}\right\|}\right) \cdot \min \left\{\left\|x_{1}-y_{1}\right\|,\left\|x_{2}-y_{2}\right\|\right\}+ \\
& +\left|\left\|x_{1}-y_{1}\right\|-\left\|x_{2}-y_{2}\right\|\right| \text {. }
\end{aligned}
$$

Taking into account the inequalities

$$
\begin{gathered}
d_{\mathbb{R}^{m}}\left(\frac{x_{1}+y_{1}}{2}, \frac{x_{2}+y_{2}}{2}\right) \leqslant d_{H}\left(\left(x_{1}, y_{1}\right),\left(x_{2}, y_{2}\right)\right), \\
d_{\mathbb{R} P^{m-1}}\left(\frac{x_{1}-y_{1}}{\left\|x_{1}-y_{1}\right\|}, \frac{x_{2}-y_{2}}{\left\|x_{2}-y_{2}\right\|}\right) \cdot \min \left\{\left\|x_{1}-y_{1}\right\|,\left\|x_{2}-y_{2}\right\|\right\} \leqslant \\
\leqslant 2 d_{H}\left(\left(x_{1}, y_{1}\right),\left(x_{2}, y_{2}\right)\right), \\
\left|\left\|x_{1}-y_{1}\right\|-\left\|x_{2}-y_{2}\right\|\right| \leqslant d_{H}\left(\left(x_{1}, y_{1}\right),\left(x_{2}, y_{2}\right)\right),
\end{gathered}
$$

we obtain

$$
\rho\left(f\left(x_{1}, y_{1}\right), f\left(x_{2}, y_{2}\right)\right) \leqslant 3 d_{H}\left(\left(x_{1}, y_{1}\right),\left(x_{2}, y_{2}\right)\right)
$$

Theorem is completed.

The set $P(X)$ of probability measures on a metric space $(X, d)$ is endowed with the Kantorovich-Rubinstein metric $d_{K R}$, [2]. The KantorovichRubinstein distance between

$$
\mu=\alpha \delta_{x}+(1-\alpha) \delta_{y}, \quad \text { and } \quad \nu=\beta \delta_{x^{\prime}}+(1-\beta) \delta_{y^{\prime}},
$$

where $\left\{x, x^{\prime}, y, y^{\prime}\right\} \in \mathbb{R}$, can be evaluated by the formula

$$
\begin{aligned}
d_{K P}(\mu, \nu)=\inf \left\{\varepsilon d\left(y^{\prime}, x\right)+(\alpha-\varepsilon) d\left(x, x^{\prime}\right)+(1-\beta-\varepsilon) d\left(y, y^{\prime}\right)+\right. & \\
& \left.+(\beta-\alpha+\varepsilon) d\left(x^{\prime}, y\right) \mid \varepsilon \geqslant 0, \varepsilon \geqslant \alpha-\beta\right\}=
\end{aligned}
$$




$$
\begin{aligned}
=\inf \{\varepsilon & \left(d\left(y^{\prime}, x\right)-d\left(x, x^{\prime}\right)-d\left(y, y^{\prime}\right)+d\left(x^{\prime}, y\right)\right)+\alpha d\left(x, x^{\prime}\right)+ \\
& \left.+(1-\beta) d\left(y, y^{\prime}\right)+(\beta-\alpha) d\left(x^{\prime}, y\right) \mid \varepsilon \geqslant 0, \varepsilon \geqslant \alpha-\beta\right\} .
\end{aligned}
$$

Lemma 3.8. Let $r>0$. For any $c>1$ and $K>0$ there exists an $r$-disjoint set $X$ in the neighborhood $O_{c r}\left(\delta_{0}\right),\left(X \subset O_{c r}\left(\delta_{0}\right)\right)$, of cardinality greater than $K$, where $\delta_{0} \in P_{2}(\mathbb{R})$.

Proof. Take

$$
X=\left\{\mu_{i}=\alpha_{i} \delta_{x_{i}}+\left(1-\alpha_{i}\right) \delta_{0} \mid x_{i}=2^{i} \cdot r, \alpha_{i}=\frac{r}{x_{i}}=2^{-i}, i \in \mathbb{N}\right\} .
$$

First we prove that $X \subset O_{c r}\left(\delta_{0}\right)$. Indeed,

$$
\begin{aligned}
d_{K R}\left(\mu_{i}, \delta_{0}\right) & =d_{K P}\left(\alpha_{i} \delta_{x_{i}}+\left(1-\alpha_{i}\right) \delta_{0}, \delta_{0}\right) \\
& =\alpha_{i} \cdot d\left(x_{i}, 0\right)=\alpha_{i} \cdot x_{i}=r<c r .
\end{aligned}
$$

Now we show that $X$ is an $r$-discrete set.

Let $j>i$, then $\alpha_{i}>\alpha_{j}$ and we see that

$$
\begin{aligned}
d_{K P}\left(\mu_{i}, \mu_{j}\right) & =d_{K P}\left(\alpha_{i} \delta_{x_{i}}+\left(1-\alpha_{i}\right) \delta_{0}, \alpha_{j} \delta_{x_{j}}+\left(1-\alpha_{j}\right) \delta_{0}\right) \\
& =\left(\alpha_{i}-\alpha_{j}\right) \cdot d\left(x_{i}, 0\right)+\alpha_{j} \cdot\left(x_{j}-x_{i}\right) \\
& =\alpha_{i} x_{i}-\alpha_{j} x_{i}+\alpha_{j} x_{j}-\alpha_{j} x_{i} \\
& =2 r-2 r \frac{x_{i}}{x_{j}}=2 r\left(1-\frac{x_{i}}{x_{j}}\right) \geqslant 2 r\left(1-\frac{1}{2}\right)=r .
\end{aligned}
$$

Therefore $X$ is countable.

Theorem 3.9. The spaces $\mathbb{R}_{+}^{3}$ and $P_{2}(\mathbb{R})$ are not coarse equivalent.

Proof. Assume that $\mathbb{R}_{+}^{3}$ and $P_{2}(\mathbb{R})$ are coarse equivalent. Then there exists a coarse uniform map $f: P_{2}(\mathbb{R}) \rightarrow \mathbb{R}_{+}^{3}$ with constant $\lambda>0$. Consider an $r$-discrete set $X \subset P_{2}(\mathbb{R})$,

$$
X=\left\{\mu_{i}=\alpha_{i} \delta_{x_{i}}+\left(1-\alpha_{i}\right) \delta_{0} \mid x_{i}=2^{i} \cdot r, \alpha_{i}=\frac{r}{x_{i}}=2^{-i}, i \in \mathbb{N}\right\} .
$$

$\mathrm{f}$ The image of the set $X$ is a $\frac{r}{\lambda}$-discrete set $f(X)$,

$$
\operatorname{diam}(f(X)) \leqslant \lambda \operatorname{diam}(X)=\lambda r,
$$

and $|f(X)|=|X|>K$ for all $K>0$. However, the cardinality of any bounded $\frac{r}{\lambda}$-discrete subset in $\mathbb{R}_{+}^{3}$ is finite. The obtained contradiction proves the theorem.

Remark 3.10. A similar result can be proved for the superextension $\lambda_{3}(\mathbb{R})$. Recall that $\lambda_{3}(\mathbb{R})$ can be defined as the quotient space of $S P^{3}(X)$ by the identification $[x, x, y] \sim[x, x, z]$. Note that $\lambda_{3}\left(S^{1}\right)$ is homeomorphic to $S^{3}$ (see [8]). 
Remark also that the spaces $\mathbb{R}_{+}^{3}$ and $P_{2}(\mathbb{R})$ are not homeomorphic. Indeed, there exists an $r$-disjoint countable set in the $c r$-neighborhood of the point $\delta_{0}$, therefore $\delta_{0}$ is not a point of local compactness. (Similar arguments cam be applied to every point $\delta_{x}$, where $\left.x \in \mathbb{R}\right)$.

Lemma 2 and Proposition 1 from [6] imply the following statement.

Corollary 3.11. There is no Lipschitz embedding of the space $P_{2}(\mathbb{R})$ into the space $\mathbb{R}^{n}$ for all $n \in \mathbb{N}$.

Let $C>0$. A sequence $x_{1}, x_{2}, \ldots, x_{n}$ in a metric space $X$ is called a $C$ chain connecting $x_{1}$ and $x_{n}$ provided $d\left(x_{i}, x_{i+1}\right) \leqslant C$ for all $i=1, \ldots, n-1$. A subset $A$ in $X$ is called asymptotically connected if there is $C>0$ such that every two points in $X$ are elements of some $C$-chain in $A$.

A subset $A$ in $X$ is called an asymptotic cut in $X$ between two sets $M_{1}, M_{2} \subset X$ if for every $C>0$ there is $r>0$ such that every $C$-chain connecting any point from $M_{1}$ and any point from $M_{2}$ necessarily intersects $B_{r}(A)$.

Let us recall that the reduced cone $C(X)$ and reduced suspension $S(X)$ of a metric space are defined in [1].

Theorem 3.12. The spaces $C\left(\mathbb{R}_{+}\right)$and $S\left(\mathbb{R}_{+}\right)$are not coarsely equivalent.

Proof. Let $i_{ \pm}: \mathbb{R}_{+} \rightarrow \mathbb{R}_{+}^{2}=\left\{(x, y) \in \mathbb{R}^{2} \mid y \geqslant 0\right\}$ be maps defined as follows: $i_{ \pm}(t)=( \pm t, 0)$. Then

$$
C\left(\mathbb{R}_{+}\right)=\mathbb{R}_{+}^{2} / i_{+}\left(\mathbb{R}_{+}\right), \quad S\left(\mathbb{R}_{+}\right)=\mathbb{R}_{+}^{2} /\left(i_{+}\left(\mathbb{R}_{+}\right) \cup i_{-}\left(\mathbb{R}_{+}\right)\right) .
$$

The metric on $C\left(\mathbb{R}_{+}\right)$and $S\left(\mathbb{R}_{+}\right)$is the quotient metric induced by the euclidean metric on $\mathbb{R}_{+}^{2}$. In the sequel we identify any subset in $\mathbb{R}_{+}^{2}$ with its quotient image image in $C(X)$ and $S(X)$.

Suppose that there exists a coarse equivalence $h: C(X) \rightarrow S(X)$.

Note that the set $i_{+}\left(\mathbb{R}_{+}\right)$is not an asymptotic cut in $C(X)$. Since the property of being an asymptotic cut is a coarse invariant, we conclude that the set $h\left(i_{+}\left(\mathbb{R}_{+}\right)\right)$is not an asymptotic cut in $S\left(\mathbb{R}^{+}\right)$.

However,

$$
\lim _{r \rightarrow \infty} d\left(i_{ \pm}\left(\mathbb{R}_{+}\right) \backslash B_{r}(0), h\left(i_{+}\left(\mathbb{R}_{+}\right)\right) \backslash B_{r}(0)\right)=\infty,
$$

and the set $h\left(i_{+}\left(\mathbb{R}_{+}\right)\right)$is asymptotically connected and unbounded. There is a neighborhood of $h\left(i_{+}\left(\mathbb{R}_{+}\right)\right)$that contains a broken line from the origin to infinity. Simple geometric arguments show that $h\left(i_{+}\left(\mathbb{R}_{+}\right)\right)$is an asymptotic cut between $i_{+}\left(\mathbb{R}_{+}\right)$and $i_{-}\left(\mathbb{R}_{+}\right)$. 


\section{REFERENCES}

[1] A. N. Dranishnikov. Asymptotic topology. Uspekhi Mat. Nauk, 55(6(336)):71-116, 2000, doi: $10.1070 / \mathrm{rm} 2000 \mathrm{v} 055 \mathrm{n} 06 \mathrm{ABEH} 000334$.

[2] L. V. Kantorovič, G. Š. Rubinšteĭn. On a space of completely additive functions. Vestnik Leningrad. Univ., 13(7):52-59, 1958.

[3] John Roe. Lectures on coarse geometry. 31:viii+175, 2003, doi: 10.1090/ulect/031.

[4] R. M. Schori. Hyperspaces and symmetric products of topological spaces. Fund. Math., 63:77-88, 1968, doi: 10.4064/fm-63-1-77-88.

[5] E. V. Ščepin. Functors and uncountable powers of compacta. Uspekhi Mat. Nauk, 36(3(219)):3-62, 255, 1981.

[6] Romanskyi M. Zarichnyi M. Asymptotic properties of the (convex) hyper-spaces. Proc. Intern. Geom. Center., 8(3-4):60-64, 2015.

[7] Romanskyi M. Zarichnyi M. Cone and join in the asymptotic categories. Visnyk of the Lviv Univ. Series Mech. Math., 83:34-41, 2017.

[8] M. M. Zarichnyı̆. The fundamental group of the superextension $\lambda_{n}(X)$, pages 24-31. Mappings and functors, Moskov. Gos. Univ., Moscow, 1984.

Mykhailo Romanskyi

Ivan Franko Drohobych State Pedagogical University, Ivana Franka St, 24, Drohobych, Lviv Region, 82100 Ukraine

Email: romanskiy.miha@ukr.net 